\title{
Nonlinearities, Smoothing, and Countercyclical Monetary Policy
}

\author{
Laura E. Jackson ${ }^{1} \quad$ Michael T. Owyang ${ }^{2}$ Daniel Soques ${ }^{3}$
}

\author{
${ }^{1}$ Bentley University \\ ${ }^{2}$ Federal Reserve Bank of St. Louis \\ ${ }^{3}$ University of North Carolina, Chapel Hill
}

July 24,2015 


\section{Disclaimer}

- The following presentation does not reflect the official opinions of the Federal Reserve Bank of St. Louis or the Federal Reserve System 


\section{Empirical Models of Monetary Policy}

- Monetary policy is a major instrument for countercyclical policy

- Much of the literature assessing the efficacy of monetary policy takes place in linear (or linearized) models

- In these models, a decrease in the fed funds rate when growth is negative has the mirror effect of an increase when growth is positive

- Responses of macro aggregates are scaled by the shock magnitudes-a 50-basis-point increase has twice the effect of a 25-basis-point increase 


\section{Nonlinear Models of Monetary Policy}

- Nonlinear models (e.g., Markov-switching models) allow for the possibility of state-dependent dynamics [Hamilton (1989)]

- This has led some to explore the effects of countercyclical policy in models with embedded cycles [e.g., Sims and Zha (2006), Ravn and Sola (2004), Owyang and Ramey (2004), Weise (1999)]

- Modeling approaches include: discrete Markov-switching (MS) with exogenous states, MS with endogenous states, threshold models, and smooth-transition models [Weise (1999)] 


\section{Nonlinearities and Countercyclical Policy}

- One criticism of the canonical MS model (e.g., the MS-VAR) is that the macro variables do not influence the state of the economy

- The probability of transitioning between regimes is constant across time

- Lowering the fed funds rate when the economy is in recession has no effect over how long the economy stays in recession

- The resulting model is quasi-linear, with the same scaling and sign symmetry effects present in purely linear models 


\section{Issues to Explore}

- Do the responses to monetary policy vary across business cycle phases?

- How do changes in monetary policy affect the probability of beginning or ending recessions?

- Is the response to countercyclical monetary policy dependent on the size (and sign) of the innovation?

- If so, does it make sense for the Fed to smooth interest rates?

- How does smoothing interest rates affect sentiment and expectations of future activity? 


\section{Sentiment within the Monetary Policy Framework}

- Previous studies have shown that uncertainty and expectations may also influence the efficacy of countercyclical policy [e.g. Galvao and Owyang (2014)]

- Monetary models, and policy rules, also tend to be forward-looking

- Smoothing in the (empirical) Taylor rule is often justified by the Fed's desire to manage expectations and overall confidence [e.g., Woodford (1999)]

- We construct a latent factor to summarize a small panel of variables meant to proxy overall sentiment and expectations of future economic dynamics 


\section{Our Approach}

- Propose a model in which the macro variables in the VAR affect the state of the economy

- Self-exciting time-varying transition probability VAR

- Changes in the state of the economy affect the dynamics

- Countercyclical policy can affect the variables within state as well as affecting the latent state

- Include a factor meant to represent consumer and producer sentiment

- Compute impulse responses that take into account the possible changes in regime 


\section{What We Find}

- Empirically relevant differences between the macroeconomic responses to contractionary and expansionary policy shocks, depending on the underlying state of the economy at the time of the shock

- Significant differences between gradual policy changes and one-time, large policy shocks 


\section{Model Overview}

- Empirical policy analysis is often conducted in linear models (i.e., VARs or linearized DSGE models that are approximated by VARs)

- We start with the baseline linear VAR to fix notation

- We then consider both a standard Markov-switching VAR and a time-varying probability VAR

- The time variation in the transition probabilities is driven by output growth, a variable in the VAR

- We include a latent factor in the TVTP-VAR meant to proxy sentiment 


\section{The Reduced-Form VAR(P)}

The canonical $\operatorname{VAR}(P)$ can be expressed in the reduced form as

$$
y_{t}=B(L) y_{t-1}+\varepsilon_{t}
$$

- $y_{t}$ represent the $N \times 1$ vector of period $-t$ variables of interest

- $\varepsilon_{t} \sim N(\mathbf{0}, \Omega)$ is the reduced-form innovation

- $\Omega$ is left unrestricted

- Any constant and trends are suppressed 


\section{The Reduced-Form VAR(P) with Regime-Switching}

Suppose we believe that the model dynamics change over the cycle:

$$
y_{t}=\left[1-S_{t}\right] B_{0}(L) y_{t-1}+S_{t} B_{1}(L) y_{t-1}+\varepsilon_{t},
$$

- $S_{t}=\{0,1\}$ follows a irreducible first-order Markov process with time-varying transition probabilities

- $p_{j i}=\operatorname{Pr}\left[S_{t}=j \mid S_{t-1}=i\right], i \in\{0,1\}$

- $\varepsilon_{t} \sim N\left(0, \Omega_{t}\right)$ with regime-dependent covariance matrix

- $\Omega_{t}=\left[1-S_{t}\right] \Omega_{0}+S_{t} \Omega_{1}$ 


\section{Time-Varying Transition Probabilities}

Suppose that the transitions depend on lags of a variable, $z_{t-d}$

$$
p_{j i}=\operatorname{Pr}\left[S_{t}=j \mid S_{t-1}=i\right]=\frac{\exp \left(\bar{\gamma}_{j i}+\gamma_{j i} z_{t-d}\right)}{\sum_{m} \exp \left(\bar{\gamma}_{j m}+\gamma_{j m} z_{t-d}\right)}
$$

- Defined for each of the regimes, $i \in\{0,1\}$, with $\sum_{m} p_{m i}=1$ for all $i$

- If, as we will hypothesize, $z$ is a transformation of variables in the vector $Y$, the system is deemed self-exciting [see, Potter (1995)]

- Shocks to the policy variable can affect the macro variables which, in turn, affect the state of the economy 


\section{Modeling Sentiment}

- Include a factor $F_{t}$ to proxy overall sentiment

- Define $Y_{t}=\left[F_{t}, y_{t}^{\prime}\right]^{\prime}$; the VAR can be rewritten as:

$$
Y_{t}=\left[1-S_{t}\right] B_{0}(L) Y_{t-1}+S_{t} B_{1}(L) Y_{t-1}+\varepsilon_{t}
$$

- $F_{t}$ summarizes the information in a panel of $M$ series, $\mathbf{X}_{t}$ :

$$
X_{m t}=\lambda_{m} F_{t}+\varsigma_{m t}, \text { for } m=1, \ldots, M
$$

- Assume that the factor provides the sole source of correlation: $\zeta_{m t} \stackrel{i i d}{\sim} N\left(0, \sigma_{m}^{2}\right)$ 


\section{Identification of Shocks and Regimes}

- Impose a within-regime causal ordering and use

Cholesky-Decomposition

- Sentiment factor ordered first and Fed funds rate ordered after output and inflation in the VAR

- Allows the macro variables and the policy rate to respond contemporaneously to shocks to overall sentiment and consumer/producer confidence

- Need additional assumptions to identify (interpret) the regimes

- Restrict $\gamma_{10}$ coefficient of the TVTP: An increase in output growth lowers the probability of switching from expansion into recession 
Data

- Monthly $\operatorname{VAR}(12)$ with the factor, the Coincident Economic Index, PCE inflation, and the Fed Funds rate

- Sample period spans 1960:1 to 2008:12, truncated to avoid issues with the ZLB

- Augment the model with the factor summarizing an unbalanced panel of sentiment data

- Conference Board Consumer Confidence Index, the University of Michigan Consumer Sentiment Index, the OECD Consumer Confidence Index, and the Institute for Supply Management Purchasing Managers Index 


\section{Estimation}

- Model parameters are estimated with the Gibbs sampler

- Sampler simulates draws from the full joint distribution by drawing from each parameter block's conditional distribution

- Employ five blocks

- VAR parameters (Normal - Inverse Wishart)

- Factor loadings and variances (Normal - Inverse Gamma)

- Transition probability parameters (Normal)

- Regimes (Hamilton Filter)

- Factors (Kalman Filter) 


\section{Posterior Regime Process}

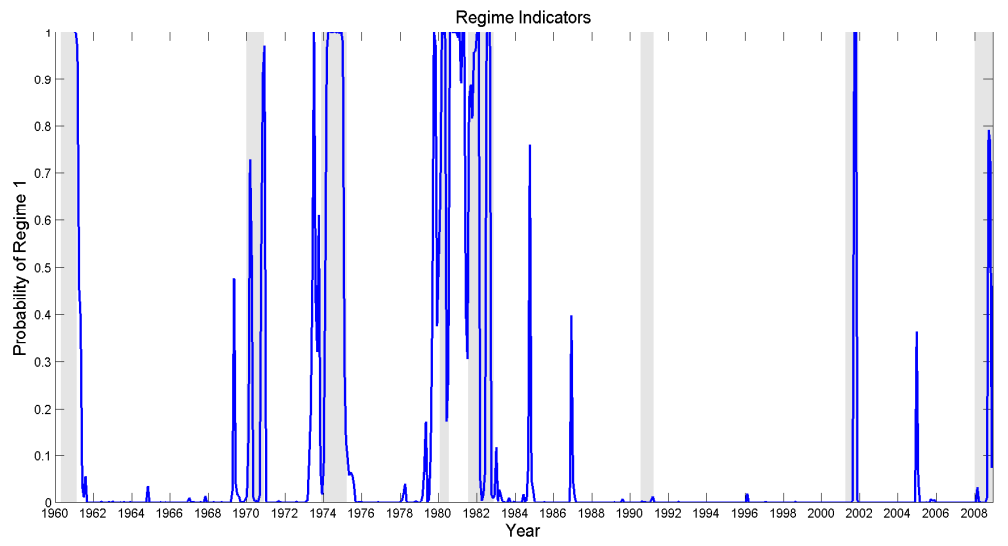




\section{Expectations Factor}

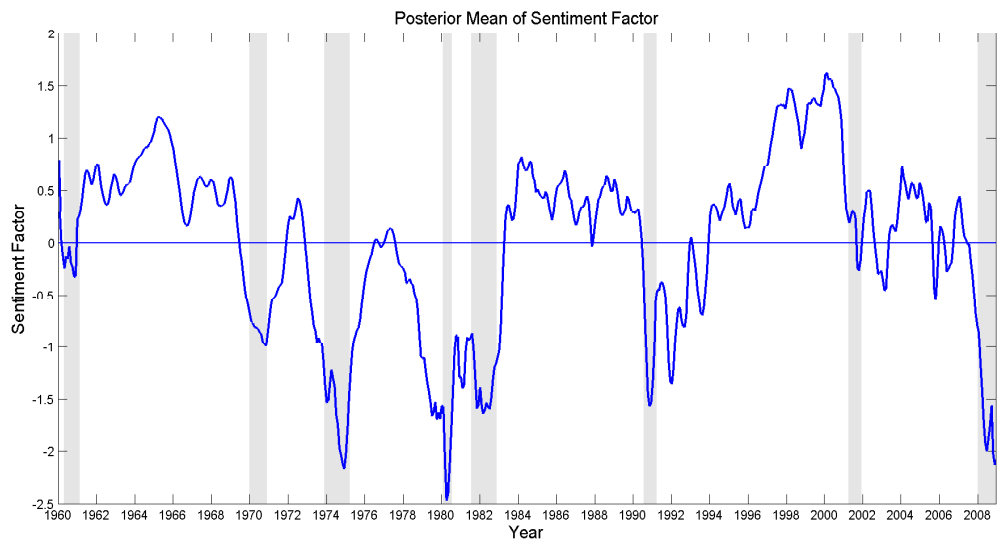




\section{Expectations Factor: MS-VAR vs. Linear VAR}

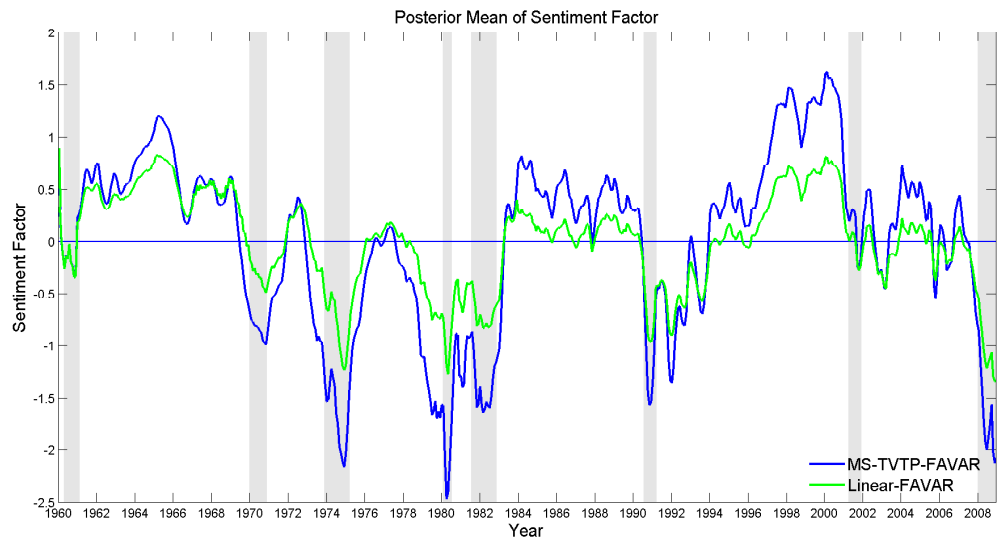




\section{Regime-Dependent Impulse Responses}

- In the standard linear model, the response to a shock is invariant to the history of the shock and the future shocks

- In MS-VARs with constant transition probabilities, the model is conditionally linear based on the regime

- Ehrmann, Ellison, and Valla (2003) established the notion of a regime-dependent response

- Responses for each regime are computed assuming that the regime does not change after the incidence of the shock 


\section{Regime-Dependent Impulse Responses}
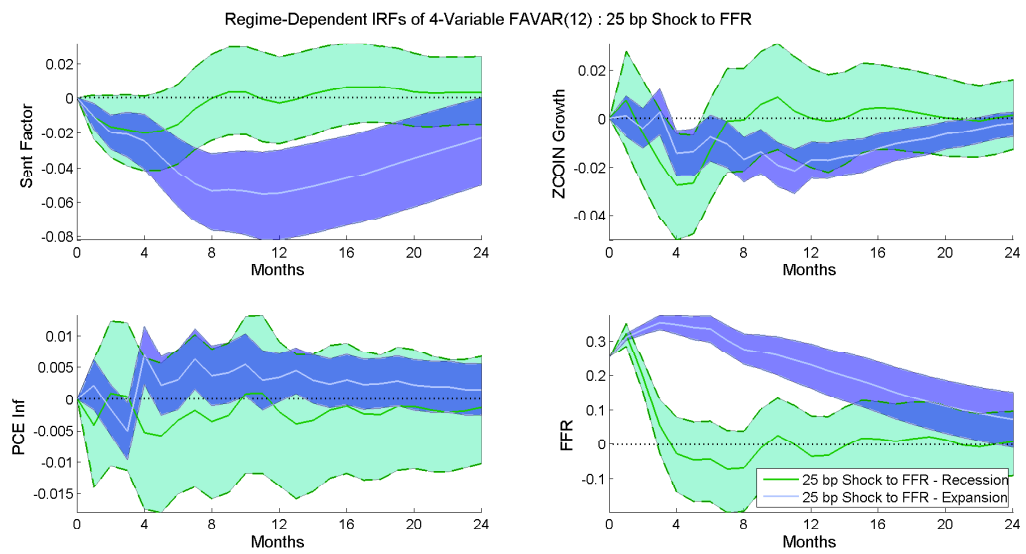


\section{Generalized Impulse Responses}

- As an alternative to regime-dependent responses, Krolzig (2006) argued that one should take into account the probability of changing regime

- Koop, Pesaran, and Potter (1998) developed the idea of generalized impulse responses

- Impulse responses can be thought of as the difference between two counterfactual expected paths

$$
\begin{aligned}
I R_{i}(h)= & E_{t}\left[Y_{t+h} \mid S_{t}=i, u_{t}=\delta,\left\{u_{t+l}\right\}_{l=1}^{h}\right] \\
& -E_{t}\left[Y_{t+h} \mid S_{t}=i, u_{t}=0,\left\{u_{t+l}\right\}_{l=1}^{h}\right]
\end{aligned}
$$

- Compute separate responses for each path beginning regime $i$

- To obtain these expectations, integrate over the history of periods with realization $S_{t}=i$

- Integrate over 100 monte carlo simulated future paths from period $t$ 


\section{Generalized Impulse Responses}

\section{Sentiment}

Contractionary Shock to FFR in Expansion

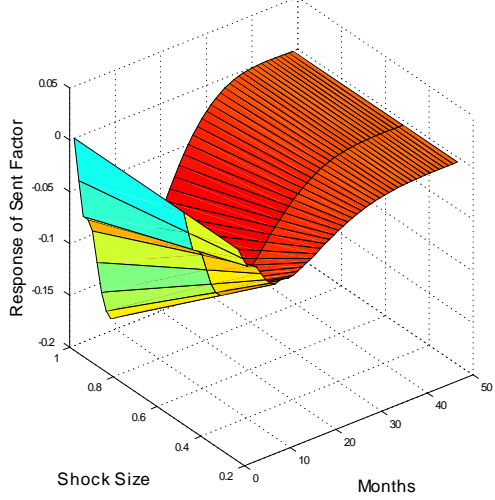

Expansionary Shock to FFR in Recession

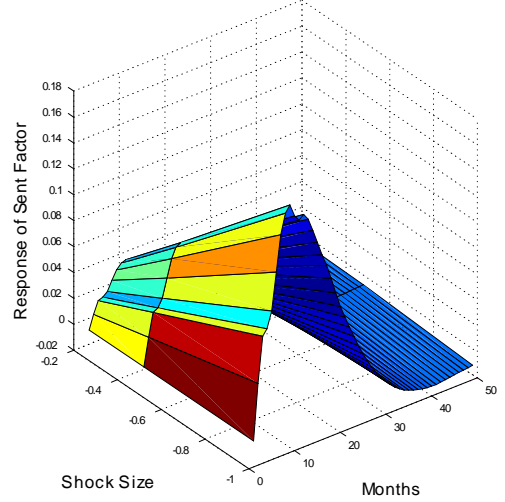




\section{Generalized Impulse Responses}

\section{ZCOIN Growth}
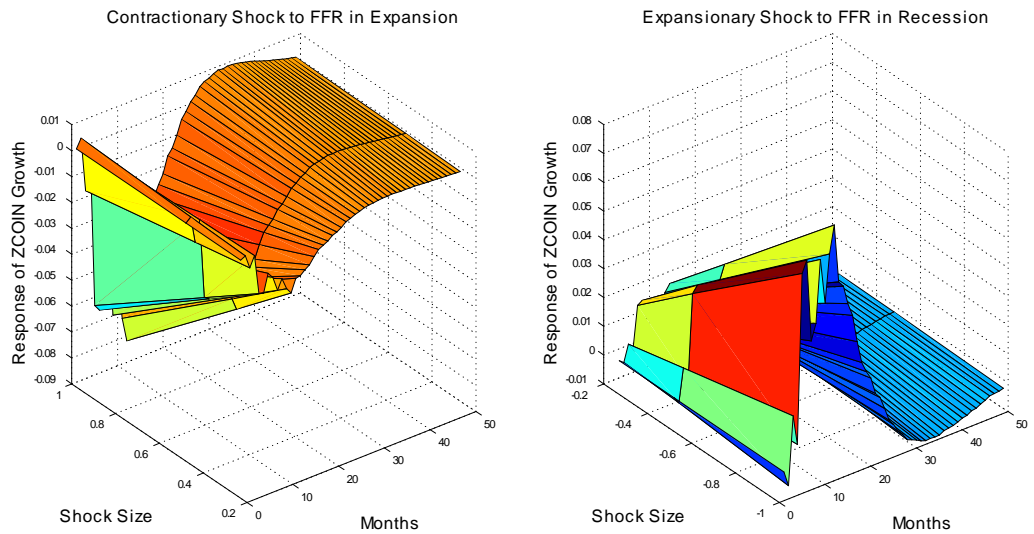


\section{Generalized Impulse Responses: Output Growth in Recession}
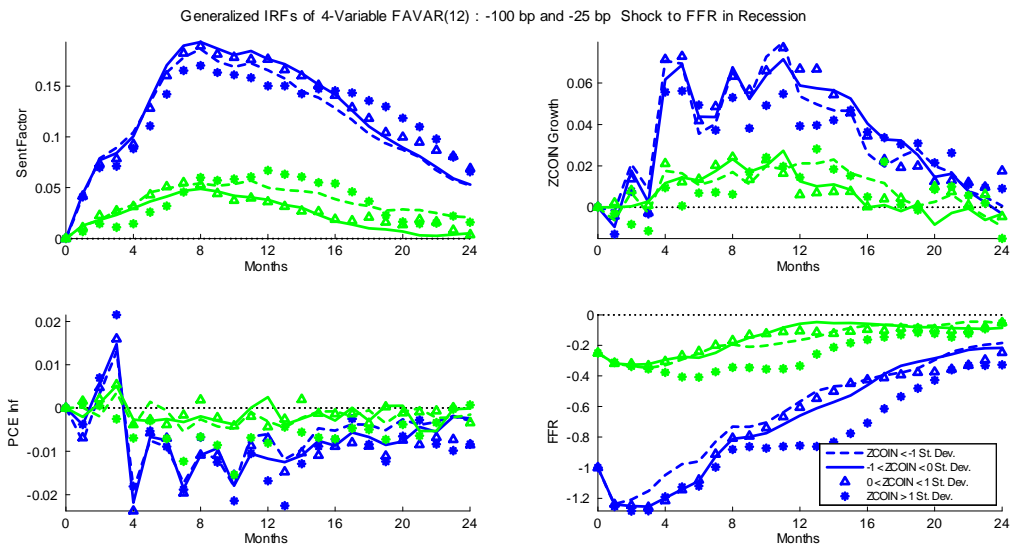


\section{Generalized Impulse Responses: Inflation in Recession}
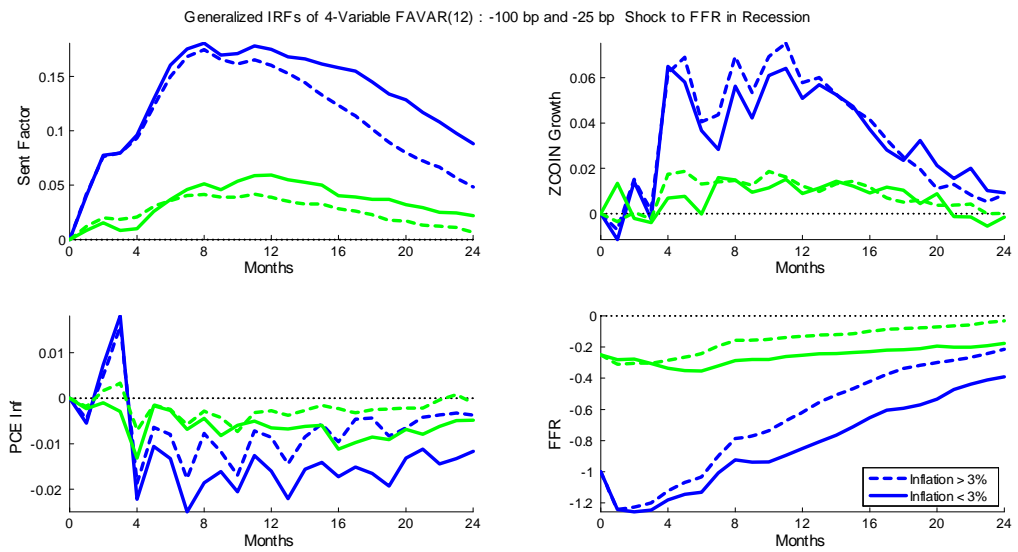


\section{The Effect of Interest Rate Smoothing}

- Empirical evidence on Taylor rules and reaction functions suggests that the Fed smooths interest rates

- Final experiment: consider the effect of interest rate smoothing in monetary policy

- For example, the Fed may anticipate that it will reduce the funds rate, say, 100 basis points in the face of a recession

- The Fed can drop the funds rate 100 basis points in one move or make a series of smaller moves

- In most cases, the Fed has opted for a smooth approach to interest rate changes

- As shown earlier, these two policies are not equivalent in a nonlinear model 


\section{The Experiment}

- Can compute the effect of compound (smoothed) policy in the GIRF framework

- Compute the path following the compound shock: $\left\{u_{t+p-1}=-25\right\}_{p=1}^{4}$

$$
Y_{t+h} \mid \mathbf{Y}_{t-1}, \Theta, S_{t}=1,\left\{u_{t+p-1}=-25\right\}_{p=1}^{4},\left\{u_{t+l}\right\}_{l=1}^{h}
$$

- Compare this to the path following a single shock: $u_{t}=-100$

$$
Y_{t+h} \mid \mathbf{Y}_{t-1}, \Theta, S_{t}=1, u_{t}=-100,\left\{u_{t+l}\right\}_{l=1}^{h}
$$

- The difference between these paths shows the effect of smoothing countercyclical policy during the recession 


\section{Responses to Compound Shocks vs. Single Large Shock}
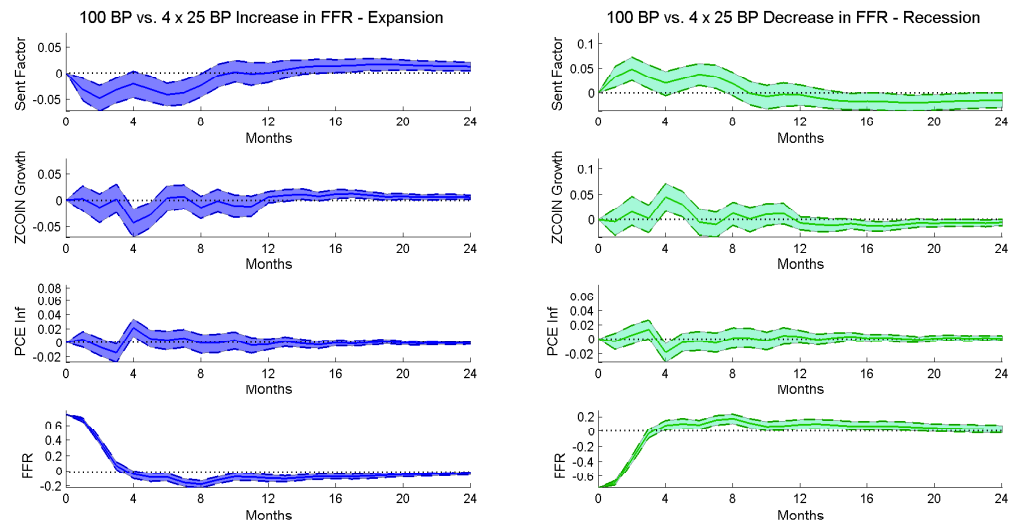


\section{Final Thoughts}

- Empirically relevant differences between the macroeconomic responses to contractionary and expansionary policy shocks, depending on the underlying state of the economy

- Framework opens up the possibility for many policy experiments

- Interest-rate smoothing

- Managing sentiment and expectations with policy

- Effects of policy in specific economic climates (inflation $>3 \%$ and output growth low, consistent periods of negative output growth, etc.) 


\section{The Structural-Form VAR}

Inference on the effect of shocks is derived from the structural form of the VAR:

$$
A^{-1} y_{t}=A^{-1} B(L) y_{t-1}+u_{t},
$$

- $u_{t} \sim N(0, \Sigma)$ are the structural shocks

- $A^{-1}$ which represents the contemporaneous effects of the structural shocks

- $A A^{\prime}=\Omega$

- $\Sigma$ is diagonal and collects the variances of the structural shocks 